\title{
Evidence of Genetic Exchange by Parasexual Recombination and Genetic Analysis of Pathogenicity and Mating Type of Parasexual Recombinants in Rice Blast Fungus, Magnaporthe oryzae
}

\author{
M. T. Noguchi, N. Yasuda, and Y. Fujita
}

First author: National Agricultural Research Center, Hokuriku Area, 1-2-1 Inada, Joetsu, 943-0193, Japan; and second and third authors: National Agricultural Research Center, Tsukuba 305-8666, Japan.

Current address of M. T. Noguchi: National Institute for Agro-Environmental Sciences, Tsukuba 305-8604, Japan.

Accepted for publication 23 February 2006.

\section{ABSTRACT}

Noguchi, M. T., Yasuda, N., and Fujita, Y. 2006. Evidence of genetic exchange by parasexual recombination and genetic analysis of pathogenicity and mating type of parasexual recombinants in rice blast fungus, Magnaporthe oryzae. Phytopathology 96:746-750.

A selectable marker gene conferring resistance to bialaphos (BI) was introduced into rice blast isolate Y90-71BI and another conferring resistance to blasticidin S (BS) into isolate 3514-R-2BS of Magnaporthe oryzae to demonstrate exchange of DNA. Colonies obtained from cocultures of these two isolates were resistant to both BI and BS and had both resistance genes as shown by Southern blot analysis of their genomic
DNA. Conidia from these BI-BS-resistant isolates had only one nucleus per cell after staining with 4',6-diamidino-2-phenylindole (DAPI). Using flow cytometry, however, these BI-BS-resistant isolates were found to be haploid. Segregation of BI-BS-resistant isolates for pathogenicity (avirulence to virulence) on rice line K59-1 was consistent with a 1:1 ratio, as was segregation for mating type. These BI-BS-resistant isolates were thus apparently derived from parasexual exchange of DNA and the segregation of pathogenicity and of mating type of the parasexual recombinants might correspond to that of the progeny of the offspring of the sexual cross.

Additional keywords: anastomosis, heterokaryosis, Oryza sativa.
Magnaporthe oryzae B. Couch (anamorph Pyricularia oryzae Cavara), previously known as $M$. grisea (Hebert) Barr (6), causes rice blast disease, which limits rice production worldwide. Resistant cultivars and fungicides usually control the disease. A vast number of breeders have developed numerous cultivars resistant to rice blast. Recently, multilines and cultivar mixtures have been used as biological and ecological methods to eliminate or reduce the need for chemicals to control many cereal diseases $(1,3,4$, $7,14,16-19,21,22,27,28,30,38)$. In Japan, many multilines of rice varieties with blast resistance have been developed, and fields planted with multilines are increasing year after year (26). However, the potential demise of the stability of the multiline system is a concern because of the potential for new pathogenic variants of the fungus to develop.

Pathogenic variants may arise via sexual mating, mutation, or parasexual recombination. Within Japan, mutation and parasexual recombination seem to have been the two major causes of variation in the pathogenicity of $M$. oryzae, because until now, ascospore production from a cross between Japanese field isolates had never been reported. Multilines, which have diverse host genotypes, are considered to produce more diverse pathogen populations than do a single line (16). Because of the benefits of induced resistance mechanism and stabilizing selection, some researchers presumed that the components of resistant and susceptible hosts toward pathogen were more durable than that of only resistant hosts in the multiline system $(3,15,22)$. Indeed, some multilines that contained resistant and susceptible hosts have been grown

Corresponding author: M. T. Noguchi; E-mail address: macha@affrc.go.jp

DOI: 10.1094/PHYTO-96-0746

(c) 2006 The American Phytopathological Society under field conditions in Japan. The multilines which include common hosts of different races in the fields may present an opportunity for the fungus to acquire new virulence genes through parasexual recombination.

Heterokaryosis and parasexual cycle have been reported in the rice blast fungus and other filamentous fungi (8-10,24,25,29, $32,33,37)$. Three processes were observed in parasexual recombination. The first process involves heterokaryosis, and heterozygous diploids occur in the second process. Heterozygous diploids are detected by the size of conidia, nuclear size, and DNA content of a nuclei. The final process is characterized by segregation and recombination during mitosis. After transient diploidy, migrating nuclei in fused cells are divided by mitotic recombination and new segregants occur. Hyphal anastomosis is a prerequisite for the establishment and development of heterokaryons. It consists of fusion between vegetatively compatible hyphae, translocation of one or more nuclei into fused cells, and compatible heterokaryotic state. Auxotrophic mutants were used to study vegetative compatibility, heterokaryosis, and parasexuality in $P$. oryzae $(5,8,9)$. However, direct evidence of the genetic exchange of DNA through parasexual recombination has not been demonstrated. Many researchers presumed that pathogenicity changes in the rice blast fungus were caused by parasexual recombination $(24,32)$, but the pathogenicity of parasexual recombinants of $M$. oryzae had not been analyzed genetically.

In this paper, selectable antibiotic-resistance genes were used to demonstrate genetic exchange of $M$. oryzae through parasexual recombination. We determined the ploidy and the number of nuclei per cell in a parasexual recombinant using flow cytometry and 4',6-diamine-2-phenylindole (DAPI)-staining of nuclei to examine if nuclei of parasexual recombinants were stable. We also genetically studied segregation of both the pathogenicity and mating type in the parasexual recombinants. 


\section{MATERIALS AND METHODS}

M. oryzae isolates. We introduced plasmid pBARKS1, a bialaphos (BI)-resistance vector containing Ignite/blast-resistance (bar) gene (2), into Y90-71 (Mat 1-1, race 102), a field isolate collected from Yunnan Province in China in 1990 and produced a BI-resistant transformant, Y90-71BI. Plasmid pBF101, a blasticidin S (BS)-resistance vector containing BSD (12), was introduced into laboratory isolate 3514-R-2 (Mat 1-2, race 136), a progeny of a cross between a field isolate, CHINOS37-1-3, collected from a lowland rice from Yunnan Province in China and identified as race 136 by N. Hayashi and field isolate FR-10, race 136, collected from South America by H. Kato, and produced a BS-resistant transformant, 3514-R-2BS. Plasmid pBF101 was provided by T. Kamakura. Y93-164a-1 (Mat1-1, race 132) and Y93-245c-2 (Mat1-2, race 137), collected from Yunnan Province in China in 1993, were used as tester isolates to determine mating type. Y90-71, 3514-R-2, Y93-164a-1, and Y93-245c-2 were hermaphroditic, female fertile, and pathogenic to rice.

Plant materials. For genetic analysis of avirulence in parasexual recombinants, one rice cultivar and one rice line were used in this study; Hattan 3 is a Japanese rice cultivar and line K59-1 is one of the $\mathrm{F}_{3}$ lines from the cross between K59 and Norin 3 (34). Norin 3 is considered to lack the resistance genes contributing to the resistance against the parental isolates used in this study. Line K59-1 has a rice blast resistance gene (Pit).

Transformation. Protoplasts of $M$. oryzae were prepared by using Novozyme 234 as described by Yelton et al. (36). Transformation of the fungus followed the method of Kimura et al. (12) with modifications. Plasmid pBARKS1 was linearized at the EcoRI recognition site, and plasmid pBF101 was linearized at the HindIII recognition site, before the transformation. To select stable transformants, 5,10 , or $20 \mu$ of the protoplast suspension was then added to $10 \mathrm{ml}$ of fresh YG1/2SC medium in collagencoated dishes $\left(100 \mathrm{~mm}\right.$ diameter) and then incubated at $28^{\circ} \mathrm{C}$. After $3 \mathrm{~h}$ of incubation, the selective reagent was added to the medium (BI at $800 \mu \mathrm{g} / \mathrm{ml}$ for isolate Y90-71 or BS at $100 \mu \mathrm{g} / \mathrm{ml}$ for isolate 3514-R-2) and further incubated for 2 days. The incubation medium was replaced every day with fresh medium with a higher concentration of the antibiotic, placing surviving colonies under the most appropriate selective pressure (isolate Y90-71 was incubated in YG medium containing BI at 200 to $800 \mu \mathrm{g} / \mathrm{ml}$, and isolate 3514-R-2 was incubated in YG medium containing BS at 50 to $100 \mu \mathrm{g} / \mathrm{ml}$ ). BI- or BS-resistant colonies thus formed were picked and transferred to potato dextrose agar (PDA) plates containing either BI $(800 \mu \mathrm{g} / \mathrm{ml})$ for isolate Y90-71 or BS $(100 \mu \mathrm{g} / \mathrm{ml})$ for isolate 3514-R-2 using forceps and further analyzed.

Coculturing. Y90-71BI and 3514-R-2BS were cocultured in $30 \mathrm{ml}$ of liquid yeast extract medium $(5 \mathrm{~g}$ of yeast extract and 1 liter of distilled water) in 100-ml flasks for 7 days, and mycelia developed were then transferred to oatmeal agar plates (50 g of oatmeal, $20 \mathrm{~g}$ of sucrose, $15 \mathrm{~g}$ of agar, and 1 liter of distilled water). After incubation for 7 days at $25^{\circ} \mathrm{C}$, hyphal fragments were collected from each mycelial mat and transferred to PDA plates containing both BI $(800 \mu \mathrm{g} / \mathrm{ml})$ and BS $(100 \mu \mathrm{g} / \mathrm{ml})$. A single colony was selected from each PDA plate. A single monoconidial isolate was then isolated from each double antibioticresistant colony.

DNA isolation and Southern blot analysis. DNA was extracted by the cetyltrimethylammonium bromide (CTAB) method (20) with modifications (35). DNA samples were digested with EcoRI (Takara Shuzo Co., Ltd., Otsu, Japan), fractionated in $0.7 \%$ agarose gel, and transferred to a Hybond-N+ membrane (Amersham, Buckinghamshire, UK). Labeling of probe DNA and hybridization were performed with the enhanced chemiluminescence (ECL) direct nucleic acid labeling/detection system (ECL system: Amersham Pharmacia Biotech, Piscataway, NJ) according to the manufacturer's recommendations and instructions.
Pot-2 repetitive sequence based-polymerase chain reaction (rep-PCR). Pot-2 rep-PCR was carried out as described by George et al. (11). Amplified PCR products were separated by gel electrophoresis in $1.0 \%$ gels in $0.5 \times$ Tris-borate-EDTA buffer.

Number of nuclei per cell. Mycelia and conidia were collected by washing a culture growing on oatmeal agar. The mycelia or a spore suspension were collected by centrifugation $(3,000 \times g$, $10 \mathrm{~min}$ ). Conidial suspension was adjusted to $10^{7}$ conidia per ml. A 1- $\mu$ l sample of the suspension was stained with DAPI (final concentration of $1 \mu \mathrm{g} / \mathrm{ml}$ in Vectashield [Vector Laboratories, Burlingame, CA]). Nuclei were observed with fluorescence microscopy using ultraviolet light.

Ploidy. Protoplasts of parental isolates and BI-BS-resistant isolates were suspended in buffer A of the Partec high resolution staining kit for plant DNA analysis (Partec GmbH, Germany). After we added $0.2 \mathrm{ml}$ of buffer A, the suspension with nuclei was filtered through a $30-\mu \mathrm{m}$ mesh filter. About $1.5 \mathrm{ml}$ of buffer B from the kit was added, and the fluorescence intensity of the nuclei was immediately measured with the Partec PA flow cytometer.

Pathogenicity. $M$. oryzae isolates were cultivated on oatmeal agar plates at $26^{\circ} \mathrm{C}$. After 11 to 12 days of subculture, aerial mycelia were washed off by gentle rubbing with a water-soaked paintbrush. The colonies were then placed under near-ultraviolet light $(360 \mathrm{~nm}, 18 \mathrm{~W})$ at $21^{\circ} \mathrm{C}$ for 3 to 4 days to induce sporulation. The conidia were scrapped and suspended in water containing $0.01 \%$ Tween 20 . The suspension was filtered through three layers of gauze mesh and adjusted to $1 \times 10^{5}$ conidia per $\mathrm{ml}$. Hattan 3 and line K59-1 were inoculated at the fourth-leaf stage by spraying $30 \mathrm{ml}$ of a spore suspension $\left(10^{5}\right.$ conidia per $\left.\mathrm{ml}\right)$ of the fungus with Diaspray (FURUPLA Co., Tokyo, Japan). Inoculated plants were immediately placed in a dew chamber at $24.5^{\circ} \mathrm{C}$ for $20 \mathrm{~h}$ and then transferred to a greenhouse at 26 to $28^{\circ} \mathrm{C}$. Six to seven days after inoculation, the pathogenicity of $M$. oryzae isolates was determined.

Mating type. Mating type was determined by crossing according to the procedure described by Yaegashi (31), using the testers.

\section{RESULTS}

Parasexual exchange. A total of 302 colonies resistant to both antibiotics were obtained. All grew on BI-BS PDA (Fig. 1).

The presence of the two antibiotic-resistance genes in BI-BSresistant isolates was then confirmed by Southern blot analysis. Forty-nine BI-BS-resistant isolates hybridized with both the probe bar gene and probe $B S D$ (Fig. 2), and 253 with either the probe bar gene or probe $B S D$.

Pot-2 rep-PCR of BI-BS-resistant isolates. Amplified bands ranged from more than 0.6 to $4.8 \mathrm{~kb}$ in length in the parental isolates and BI-BS-resistant isolates (Fig. 3). Pot-2 rep-PCR fingerprints distinguished the parental isolates and fingerprint patterns of BI-BS-resistant isolates composed of bands from parental isolates.

Number of nuclei per cell. Hyphal cells were too small to distinguish each cell and we were unable to determine the number of nuclei per cell. DAPI staining showed the presence of three nuclei in each conidium of parental isolates and BI-BS-resistant isolates. Conidia of the rice blast fungus usually consisted of three cells. The conidia of parental isolates and BI-BS-resistant isolates were mostly uninucleate (Fig. 4). Some had two nuclei, but these seemed to be in the process of cell division.

Ploidy. The flow cytometry histograms of BI-BS-resistant isolates and the parental isolates were nearly identical. They had three peaks, but the peak near zero resulted from debris in the nuclear DNA. The peaks represented intensity of fluorescence. The peak positions corresponded to the ploidy level; the first peaks represented the haploid and the second represented the diploid. The position of the first peak was about 65.2 in Y90-71. The first peaks were located at $67.58,61.73,61.85$, and 64.93 in 
3514-R-2BS, BI-BS-resistant isolates (BIS1, BIS132, and BIS209). The second peaks were located at 121.85, 123.93, 118.25, 117.03, and 119.27 in Y90-71BI, 3514-R-2BS, BIS1, BIS132, and BIS209. The second peak was located about the same distance from the first peak in each pattern, indicating that cells were in the G2 period in the cell cycle. From our results, the ploidy of BIBS-resistant isolates was haploid, as was the case with the parental isolates.

Segregation of avirulence/virulence in BI-BS-resistant isolates. Y90-71 has AvrHattan3 and was avirulent on cv. Hattan 3, but it was virulent on line K59-1 carrying Pit. Isolate 3514-R-2 has AvrPit and was virulent on Hattan 3, but it was avirulent on line K59-1. Inoculation of Hattan 3 with the 49 BI-BS-resistant isolates revealed that 12 isolates were avirulent and 37 were virulent. A chi-square value of 12.76 showed that the segregation was not consistent with a 1:1 ratio (Table 1). Inoculation of line K59-1 with the 49 BI-BS-resistant isolates yielded a segregation ratio of 24 avirulent to 25 virulent isolates. A chi-square value of 0.02 indicated that the segregation was consistent with a 1:1 ratio.

Segregation of mating. A total of 21 of the 49 BI-BS-resistant isolates were of Mat1-1 and 28 were of Mat1-2. A chi-square

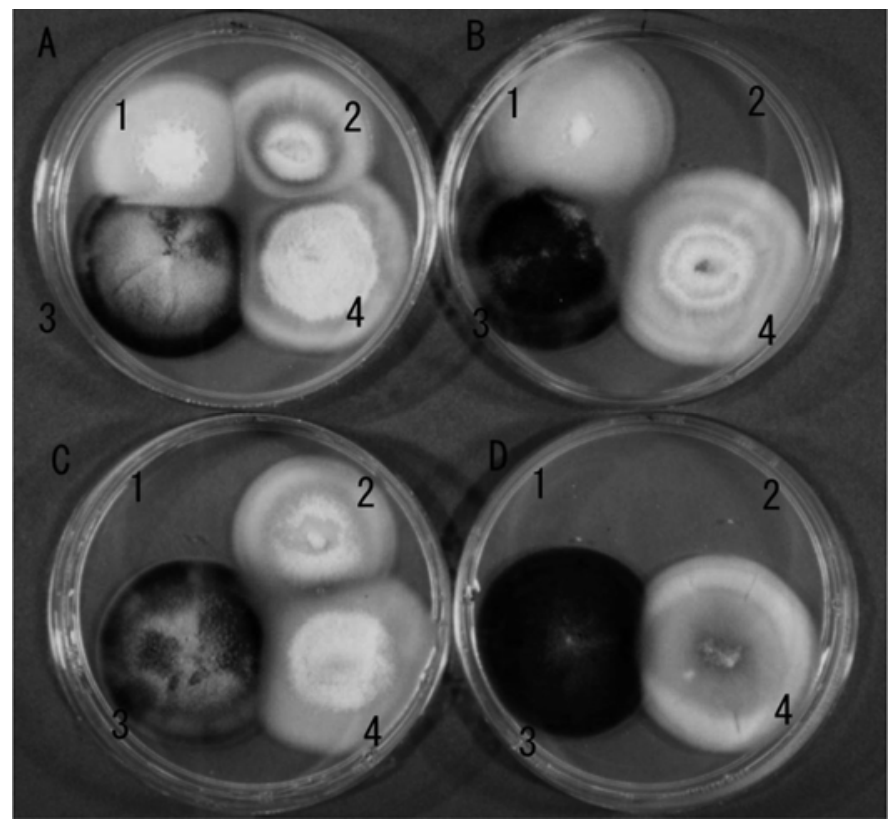

Fig. 1. Colonies of parental isolates and BI-BS-resistant isolates on potato dextrose agar (PDA) and selective medium. A, PDA; B, PDA with BI (800 $\mu \mathrm{g} / \mathrm{ml})$; C, PDA with BS $(100 \mu \mathrm{g} / \mathrm{ml})$; and D, PDA with BI $(800 \mu \mathrm{g} / \mathrm{ml})$ and BS (100 $\mu \mathrm{g} / \mathrm{ml}) .1$, Y90-71BI (parent); 2, 3514-R-2BS (parent); and 3 and 4, BI-BS-resistant isolates (BIS1, BIS2). value of 1.00 indicated that segregation was consistent with a 1:1 ratio $(P=0.3$ to 0.5$)$.

\section{DISCUSSION}

The generation of genetic diversity in plant pathogens is a critical problem for the control of plant diseases. One of the significances of parasexuality in asexual filamentous fungi is to produce genetic diversity (37). We have provided the first direct evidence on genetic exchange of DNA through parasexual recombination in $M$. oryzae by using two different antibiotic-resistance

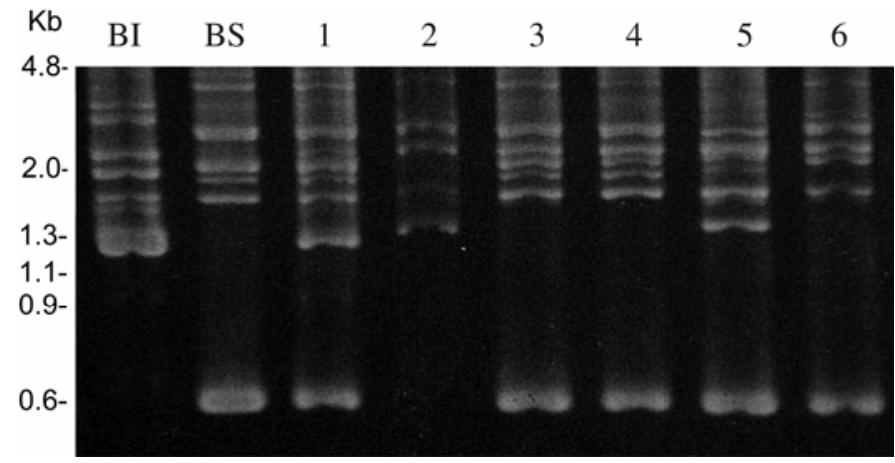

Fig. 3. Agarose gel electrophoresis of Pot-2 rep-PCR fingerprint patterns from genomic DNA of parental isolates and BI-BS-resistant isolates. BI, Y90-71BI (parent); BS, 3514-R-2BS (parent); the lanes 1 to 6, BI-BS-resistant isolates (lane 1, BIS71; 2, BIS110; 3, BIS112; 4, BIS116; 5, BIS120; and 6, BIS122).

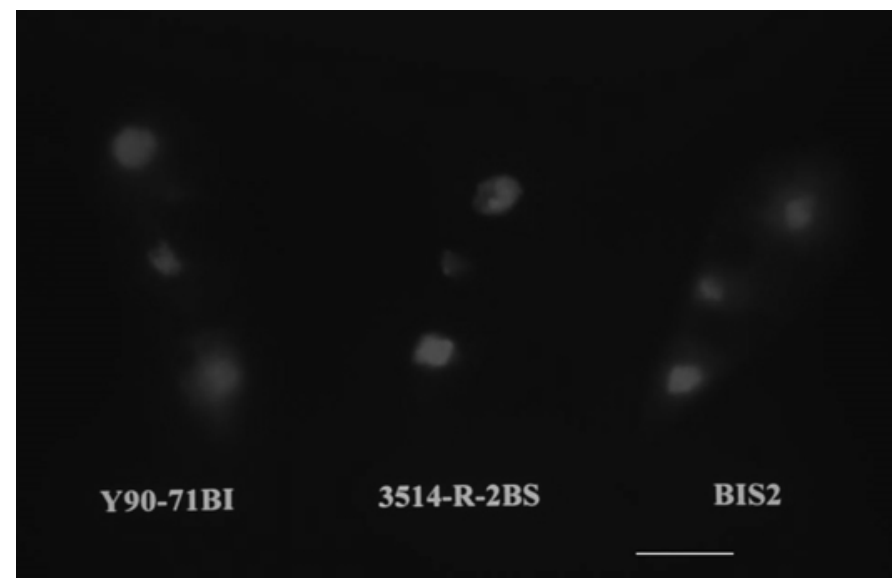

Fig. 4. 4',6-Diamidino-2-phenylindole (DAPI) staining of conidia of Magnaporthe oryzae. Y90-71BI (parent), 3514-R-2BS (parent), and BIS2 (BI-BS resistant isolate). Scale bar indicates $10 \mu \mathrm{m}$.
A

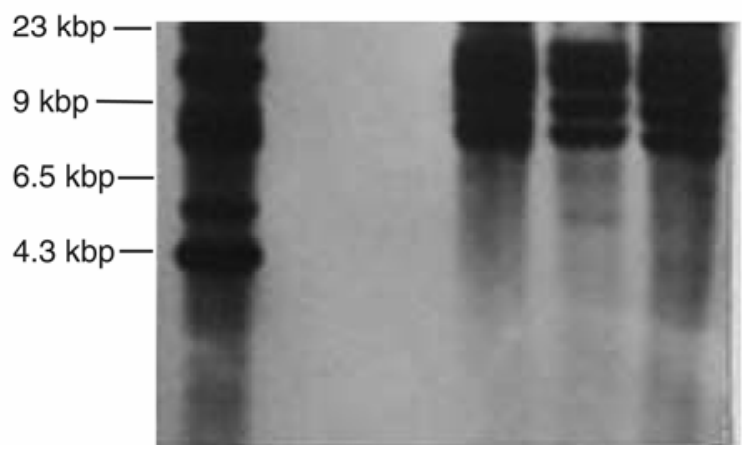

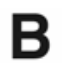

$\mathrm{BI} \quad \mathrm{BS}$

$\mathrm{BISI}$

BIS2 BIS3

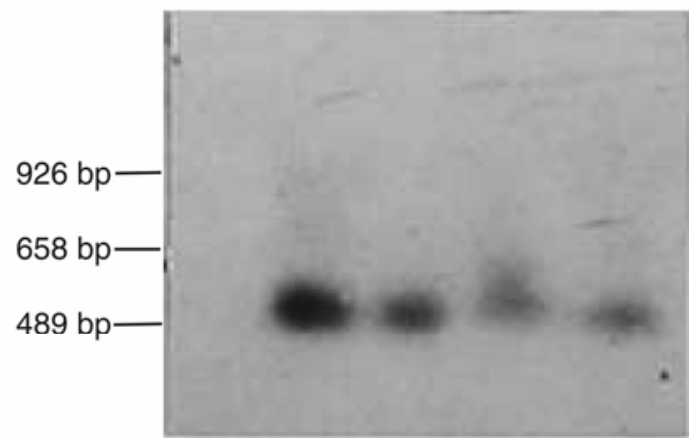

Fig. 2. Southern blot analysis of parental isolates and BI-BS-resistant isolates. Total genomic DNA was digested with EcoRI. Electrophoresed DNA digests were hybridized with the A, probe bar gene B, probe BSD. BI, Y90-71BI (parent); BS, 3514-R-2BS (parent); and BI-BS-resistant isolates (BIS1, BIS2, BIS3). 
genes. BI-BS-resistant isolates proved to have different phenotypes from the parental isolates in virulence and mating type.

A majority (85\%) of BI-BS-resistant isolates had, however, either of the two antibiotic-resistance genes; they did not originate from parasexual recombination. They seem to have acquired resistance through either mutation or cross feeding. BS is used as a fungicide to control rice blast, and BS-resistant mutants have been known in nature. We considered that the isolates were able to acquire resistance to this fungicide. BI is used as a herbicide, and in nature, BI-resistant mutants have not been known in this fungus. However, the fungus could have acquired resistance to the herbicide. Even though mutation to pesticides is considered to be involved in BI-BS-resistant isolates, its rate seems too high. Cross feeding possibly contributed to the phenomenon also.

The hyphal cells of filamentous fungi are either uninucleate or multinucleate. It was reported that hyphal and conidial cells of $P$. oryzae are predominately uninucleate and haploid (H. Saito and S. Kato, unpublished data). Yamasaki and Niizeki (32) reported that the majority of cells in conidia, germ tubes, mycelia, and conidiophores of $P$. oryzae are uninuclear, based on results of nuclear staining with Giemsa's solution, basic fuchsin, acetoorcein, or aceto-carmine.

The ploidy level in fungal cells has in the past been determined by quantifying the DNA with a colorimetric assay such as the Feulgen reaction, by physical methods such as meiotic chromosome counts, and by spore size. Flow cytometry is a convenient method for estimating the amount of DNA in a nucleus and for determining ploidy in fungi, plant, and mammal cells. To our knowledge, this study is the first report on the determination of ploidy of parasexual recombinants in $M$. oryzae by flow cytometry. The conidia of the parental isolates and of BI-BS-resistant isolates were almost always uninucleate and had the same ploidy. These results suggested that the isolates derived from parasexual recombination were stable as were the parental isolates.

Yasuda et al. (35) reported that the segregation of avr/vir in the progeny derived from crossing Y90-71 and 3514-R-2 on Hattan 3 and on line K59-1 (data not shown) fitted a 1:1 ratio. The discrepancy in the segregation of virulence to avirulence of BIBS-resistant isolates on Hattan 3 from the 1:1 ratio was assumed that the antibiotic-resistance genes and genes for virulence to Hattan 3 might be linked as a result of the transformation. Another reason was that the segregation of virulence of the parasexual recombinants in the rice blast fungus was not corresponding to that of sexual progeny. However, from the results of the segregation in virulence on K59-1 and in mating type, the genetic characteristics of parasexual recombinants were shown to segregate as does the sexual progeny. Genetic studies of parasexual recombinants are further needed.

Heterokaryon and parasexuality have been known to occur in laboratory cultures of the rice blast fungus (8-10), and studies on lineage of isolates from the Indian Himalayans and Philippines $(13,37)$ suggested the occurrence of parasexual recombination in nature indirectly, but parasexuality under field condition has never been observed. The potential of hyphal fusion was considered to be the reason for possible parasexual recombination. Hyphal anastomosis between isolates from the same locality was reported in the rice blast fungus $(5,37)$.

TABLE 1. Segregation of avirulence (Avr) to virulence (Vir) in BI-BSresistant isolates derived from co-culturing Y90-71BI and 3514-R-2BS

\begin{tabular}{lccccc}
\hline & \multicolumn{2}{c}{ Parental isolates $^{\mathrm{a}}$} & \multicolumn{2}{c}{ Isolates } & \\
\cline { 2 - 3 } Rice & Y90-71BI & 3514-R-2BS & Avr:Vir & $\chi^{2} 1: 1$ & $P$ value \\
\hline Hattan 3 & $\mathrm{R}$ & $\mathrm{S}$ & $12: 37$ & 12.76 & $<0.01$ \\
Line K59-1 & $\mathrm{S}$ & $\mathrm{R}$ & $24: 25$ & 0.02 & $0.80-0.90$ \\
\hline
\end{tabular}

${ }^{a} \mathrm{R}$, resistant reaction; $\mathrm{S}$, susceptible reaction.
Frequency of parasexual recombination is a critical issue, but its exact estimate has not been reported even in laboratory experiments. Methods for efficient production of parasexual recombinants and their selection need to be developed to examine the rate of parasexual recombination in the laboratory. Characteristics concerning the stability and fitness of parasexual recombinants are important for their survive under field conditions. Results from nuclei observation and ploidy determination imply the stability of parasexual recombinants in nature. Their behavior in the field further remains to be studied even though a few reports have dealt with the fitness of parasexual recombinants (23). Parasexuality is thought to be useful for genetic diversification in rice blast fungus populations lacking sexual production. Genetic exchange thorough parasexual recombination would occur and contribute to the survival under severe condition, in addition to generating a broad pathogenic spectrum against rice cultivars.

A rice multiline having diverse host genotypes in blast resistance gene may support different parasitic genotypes (different races) (16). Our results suggest the occurrence of complex races of the rice blast fungus through parasexual exchange in multiline fields. Their fitness should further be compared in different paddy fields planted under different multiline systems so that the system may operate effectively to control rice blast.

\section{ACKNOWLEDGMENTS}

We thank N. Hayashi for providing fungal isolates, K. Tachibana for BI and T. Kamakura for the plasmid vector. We appreciate the technical advice of N. Ohmido on DAPI staining and of T. Takai on flow cytometer. We thank N. Matsumoto for critical review of the manuscript.

\section{LITERATURE CITED}

1. Abbott, D. C., Burdon, J. J., Brown, A. H. D., Read, B. J., and Bittisnich, D. 2000. The incidence of barley scald in cultivar mixtures. Aust. J. Agric. Res. 51:355-360.

2. Avalos, J., Geever, R. F., and Case, M. E. 1989. Bialaphos resistance as a dominant selectable marker in Neurospora crassa. Curr. Genet. 16:369372 .

3. Browning, J. A., and Frey, K. J. 1969. Multiline cultivars as a means of disease control. Annu. Rev. Phytopathol. 7:355-382.

4. Chin, K. M., and Wolfe, M. S. 1984. The spread of Erysiphe graminis f. sp. hordei in mixtures of barley varieties. Plant Pathol. 33:89-100.

5. Correll, J. C., Harp, T. L., Guerber, J. C., Zeigler, R. S., Liu, B., Cartwright, R. D., and Lee, F. N. 2000. Characterization of Pyricularia grisea in the United States using independent genetic and molecular markers. Phytopathology 90:1396-1404.

6. Couch, B. C., and Kohn, L. M. 2002. A multilocus gene genealogy concordant with host preference indicates segregation of a new species, Magnaporthe oryzae, from M. grisea. Mycologia 94:683-693.

7. Cowger, C., and Mundt, C. C. 2002. Effects of wheat cultivar mixtures on epidemic progression of Septoria tritici blotch and pathogenicity of Mycosphaerella graminicola. Phytopathology 92:617-623.

8. Crawford, M. S., Chumley, F. G., Weaver, C. G., and Valent, B. 1986. Characterization of the heterokaryotic and vegetative diploid phases of Magnaporthe grisea. Genetics 114:1111-1129.

9. Fatemi, J., and Nelson, R. R. 1978. Inter-isolate heterokaryosis in Pyricularia oryzae. Phytopathology 68:1791-1794.

10. Genovesi, A. D., and Magill, C. W. 1976. Heterokaryosis and parasexuality in Pyricularia oryzae Cavara. Can. J. Microbiol. 22:531-536.

11. George, M. L. C., Nelson, R. J., Zeigler, R. J., and Leung, H. 1998. Rapid population analysis of Magnaporthe grisea by using rep-PCR and endogenous repetitive DNA sequences. Phytopathology 88:223229.

12. Kimura, M., Izawa, K., Yoneyama, K., Arie, T., Kamakura, T., and Yamaguchi, I. 1995. A novel transformation system for Pyricularia oryzae: Adhesion of regenerating fungal protoplasts to collagen-coated dishes. Biosci. Biotech. Biochem. 59:1177-1180.

13. Kumar, J., Nelson, R. J., and Zeigler, R. S. 1999. Population structure and dynamics of Magnaporthe grisea in the Indian Himalayas. Genetics 152:971-984.

14. Manthey, R., and Fehrmann, H. 1993. Effect of cultivar mixtures in wheat on fungal diseases, yield and profitability. Crop Prot. 12:63-68.

15. Marshall, D. R. 1977. The advantages and hazards of genetic homogeneity. Ann. NY Acad. Sci. 287:1-20. 
16. Mundt, C. C. 2002. Use of multiline cultivars and cultivar mixtures for disease management. Annu. Rev. Phytopathol. 40:381-410.

17. Mundt, C. C., Brophy, L. S. and Schmitt, M. S. 1995. Choosing crop cultivars and cultivar mixtures under low versus high disease pressure: A case study with wheat. Crop Prot. 14:509-515.

18. Mundt, C. C., and Browning, J. A. 1985. Development of crown rust epidemics in genetically diverse oat populations: Effect of genotype unit area. Phytopathology 75:607-610.

19. Mundt, C. C., and Leonard, K. J. 1986. Analysis of factors affecting disease increase and spread in mixtures of immune and susceptible plants in computer-simulated epidemics. Phytopathology 76:832-840.

20. Murray, M. G., and Thompson, W. F. 1980. Rapid isolation of high molecular weight plant DNA. Nucleic Acids Res. 8:4321-4325.

21. Nakajima, T., Sonoda, R., and Yaegashi, H. 1996. Effect of a multiline of rice cultivar Sasanishiki and its isogenic lines on suppressing rice blast disease. Ann. Phytopathol. Soc. Jpn. 62:227-233.

22. Nakajima, T., Sonoda, R., Yaegashi, H., and Saito, H. 1996. Factors related to suppression of leaf blast disease with a multiline of rice cultivar Sasanishiki and its isogenic lines. Ann. Phytopathol. Soc. Jpn. 62:360364.

23. Namai, T., Ehara, Y., and Togashi, J. 1990. Changes in aggressiveness of a Pyricularia oryzae isolate (race 337) by successive passage on rice cultivars with different true resistance gene. Ann. Phytopathol. Soc. Jpn. 56:1-9.

24. Namai, T., and Yamanaka, S. 1982. Studies on variation in virulence of rice blast fungus, Pyricularia oryzae Cavara I. Variant formation by the Paring-cultivation and -inoculation of two different pathogenic isolates. Ann. Phytopathol. Soc. Jpn. 48:466-470.

25. Papa, K. E. 1978. The parasexual cycle in Aspergillus parasiticus. Mycologia 70:766-773.

26. Sasaki, T., Abe, S., Matsunaga, K., Okamoto, E., Nagano, K., Tanno, K., Chiba, Y., Kano, A., Uematsu, K., Takizawa, H., Hayasaka, H., Wakui, S., Kuroda, T., Usuki, S., Chiba, B., Miyano, N., Sasaki, K., and Endo, T. 2002. Multiline rice variety of Sasanishiki "Sasanishiki BL". Bull. Miyagi Pref. Furukawa Agric. Exp. Stn. 3:1-35.

27. Wilson, J. P., Gates, R. N., and Panwar, M. S. 2001. Dynamic multiline population approach to resistance gene management. Phytopathology 91:255-260.

28. Wolfe, M. S. 1985. The current status and prospects of multiline cultivars and variety mixtures for disease resistance. Annu. Rev. Phytopathol. 23:251-273.

29. Xia, J. Q., and Correll, J. C. 1995. Examination pf mitotic stability and hybridization potential between two genetically distinct haplotypes of Magnaporthe grisea. Exp. Mycol. 19:171-177.

30. Xu, X.-M., and Ridout, M. S. 2000. Stochastic simulation of the spread of race-specific and race-nonspecific aerial fungal pathogens in cultivar mixtures. Plant Pathol. 49:207-218.

31. Yaegashi, H. 1981. Studies on the perfect stage of Pyricularia species. Bull. Tohoku Natl. Agric. Exp. Stn. 63:49-125.

32. Yamasaki, Y., and Niizeki, H. 1965. Studies on variation of the rice blast fungus, Pyricularia oryzae Cav. I. Karyological and genetical studies on variation. Bull. Natl. Inst. Agric. Sci. Jpn. 13:231-273.

33. Yang, H. A., Tommerup, I. C., Sivasithamparam, K., and O'Brien, P. A 1992. Heterokaryon formation with homokaryons derived from protoplasts of Rhizoctonia solani anastomosis group eight. Exp. Mycol. $16: 268-278$

34. Yasuda, N., Fujita, Y., and Noguchi, M. 2004. Identification of avirulence genes in the rice blast fungus corresponding to three resistance genes in Japanese differentials. J. Gen. Plant Pathol. 70:202-206.

35. Yasuda, N., Noguchi, M. T., and Fujita, Y. 2005. Identification of an avirulence gene in the fungus Magnaporthe grisea corresponding to a resistance gene at the Pik locus. Phytopathology 95:768-772.

36. Yelton, M. M., Hamer, J. E., and Timberlake, W. E. 1984. Transformation of Aspergillus nidulans by using a $\operatorname{trpC}$ plasmid. Proc. Natl. Acad. Sci. USA 81:1470-1474.

37. Zeigler, R. S., Scott, R. P., Leung, H., Bordeos, A. A., Kumar, J., and Nelson, R. J. 1997. Evidence of parasexual exchange of DNA in the rice blast fungus challenges its exclusive clonality. Phytopathology 87:284294.

38. Zhu, Y., Chen, H., Fan, J., Wang, Y., Li, Y., Chen, J., Fan, J., Yang, S., Hu, L., Leung, H., Mew, T. W., Teng, P. S., Wang, Z., and Mundt, C. C. 2000. Genetic diversity and disease control in rice. Nature 406:718-722. 